\title{
Micelles of Pulmonary Surfactant in Human Amniotic Fluid at Term
}

\author{
KOJI NISHIJIMA, KEN-ICHI SHUKUNAMI, HIROKAZU TSUKAHARA, MAKOTO ORISAKA, JUN'ICHIRO MIURA, AND \\ FUMIKAZU KOTSUJI
}

\author{
Department of Obstetrics and Gynecology [K.N., KS, MO, F.K.], Department of Pediatrics [H.T.], Department of Applied Chemistry and \\ Biotechnology [J.M.], University of Fukui, Fukui, 910-1193, Japan
}

\begin{abstract}
Studies using in vitro analysis have shown that the interaction between pulmonary surfactant and vernix caseosa could explain the appearance of amniotic fluid turbidity. That phenomenon is interpreted based on the "roll-up" hypothesis. We tested the roll-up hypothesis by examining the presence of micelles of pulmonary surfactant in human amniotic fluid at term. Amniotic fluid samples were collected from each of six healthy pregnant women at term and at $16 \mathrm{wk}$ of gestation. These samples were stained negatively and analyzed using an electron microscope. Ultrastructures present in amniotic fluid were compared with the structure of micelles derived from suspended surfactant TA isolated from bovine lung. Surfactant TA formed spheroidal and rod-shaped micelles $10-70 \mathrm{~nm}$ in diameter above the critical micelle concentration. Identical micelle particles were described in human amniotic fluid at term. In addition, surfactant protein B was identified in the micelle fraction of amniotic fluid. However, no micelles were found in human amniotic fluid taken at 16 wk of gestation. Our results support the view that pulmonary surfactant could induce the detachment of vernix caseosa and increase the turbidity of the amniotic fluid. (Pediatr Res 60: 196-199, 2006)
\end{abstract}

$\mathrm{T}$ The turbidity of the amniotic fluid increases with gestational age during the third trimester (1-5). Studies using in vitro analysis have shown that the interaction between pulmonary surfactant and vernix caseosa could explain the appearance of amniotic fluid turbidity (5). Narendran et al. (5) speculated that pulmonary surfactant induces a "roll-up" phenomenon that leads to detachment of vernix caseosa from the fetal skin surface. Droplets of oil rise from the oil/substrate interface when the surfactant displaces the oily masses from the substrate in aqueous media. This displacement is called "roll-up" and is strictly controlled by micellization process kinetics $(6,7)$.

Surfactants are chemical amphipathic compounds; they contain both hydrophobic and hydrophilic ends (8). They form micelles according to their $\mathrm{CMC}$ in an aqueous environment

Received September 20, 2005; accepted March 23, 2006

Correspondence: Koji Nishijima, M.D., Department of Obstetrics and Gynecology, University of Fukui, Matsuoka-cho, Yoshida-gun, Fukui 910-1193, Japan; e-mail: kojigyne@fmsrsa.fukui-med.ac.jp

This work was supported in part by a Grant-in-Aid for Scientific Research No. 15659392 from the Ministry of Education, Culture, Sports, Science, and Technology, Japan. The sponsor had no role in the study design, data collection, data analysis, or writing of the report.

DOI: 10.1203/01.pdr.0000228329.90502.3a-
(8). Pulmonary surfactant, a mixture of specific phospholipids and apoproteins, is a natural surface-active agent $(1,2)$. Pulmonary surfactant could thereby form micelles in human amniotic fluid. Numerous studies using electron microscopy have revealed pulmonary surfactant in amniotic fluid as a storage medium: lamellar body $(2,3)$. However, to our knowledge, no studies have investigated micelles of pulmonary surfactant.

To test the roll-up hypothesis proposed by Narendran et al. (5), we used electron microscopy to determine the presence of micelles of alveolar surfactant in human amniotic fluid at term. Since impurities in amniotic fluid disturb ultrastructural analysis, we first analyzed micelles derived from surfactant TA (Surfacten; Mitsubishi Pharma Corp., Osaka, Japan) for pattern recognition. We then analyzed mixtures of surfactant TA and vernix caseosa and samples of amniotic fluid collected at term or at 16 wk of gestation. We also performed experiments to show that a specific component of the surfactant, $\mathrm{SP}-\mathrm{B}$, is associated with the micelles, and that micelles derived from human amniotic fluid induce the detachment of vernix caseosa under in vitro condition.

\section{MATERIALS AND METHODS}

Preparation of surfactant TA. The composition of surfactant TA, which is isolated from bovine lung and used clinically (9), is similar to Survanta (Ross Laboratories, Columbus, OH). It comprises $83.5 \%$ phospholipids, $7.0 \%$ FFA, $7.0 \%$ triglycerides, $1.0 \%$ hydrophobic surfactant-associated protein, and other components (10). First, we determined the CMC of surfactant TA in a physiologic saline solution by measuring its surface tension with a Wilhelmytype surface-tension meter (CBVP A-1; Kyowa Interface Science Co., Tokyo, Japan). A stock solution of surfactant TA was prepared by dissolving $108 \mathrm{mg}$ of surfactant TA into saline and diluting it to $50 \mathrm{~mL}$ with saline solution. The stock solution was diluted accurately to determine the surface tension of surfactant TA. The value of the CMC of surfactant TA was obtained from the concentration/surface-tension curve $(11,12)$. Surfactant TA was suspended in a physiologic saline solution at $37^{\circ} \mathrm{C}$ to analyze micelles. The surfactant TA solution was adjusted with a phospholipid concentration of $25,50,75,100$, 150,200 , or $500 \mu \mathrm{g} / \mathrm{mL}$. Samples were maintained at $37^{\circ} \mathrm{C}$ until electron microscopic analysis.

Preparation of vernix caseosa-surfactant TA mixture. Samples were prepared according to the procedure of Narendran et al. (5). Briefly, vernix caseosa was harvested from full-term infants who were free of any antenatal complications, and stored in sterile vials at the time of delivery. Samples were refrigerated at $-80^{\circ} \mathrm{C}$ until experiments. We applied $10 \mathrm{mg}$ of vernix caseosa pooled from three term infants to the interior walls of $1.5-\mathrm{mL}$ polypropylene microfuge tubes and spread it on the wall using a glass pestle to form an even

Abbreviations: CMC, critical micelle concentration; SP-B, surfactant protein $\mathrm{B}$ 
coating. Then, $1 \mathrm{~mL}$ of normal saline with $150 \mu \mathrm{g}$ phospholipid derived from surfactant TA was added to the tubes. The mixture was agitated gently by slow rotation on a rotisserie/shaker (Labquake; Barnstead/Thermolyne Corp., Dubuque, IA). The rotisserie was placed overnight in a thermally equilibrated room at $37^{\circ} \mathrm{C}$. The liquid was decanted and subsequently analyzed electron microscopically.

Preparation of amniotic fluid samples. Amniotic fluid samples were collected from six healthy Japanese women at term ( $\geq 37$ wk gestation) singleton pregnancies. All six had been scheduled for elective cesarean delivery. In addition, we studied six healthy pregnant women at 16 wk of gestation who had been scheduled for transabdominal amniocentesis for fetal chromosome analysis. Patients were excluded if they had a chronic or acute physical illness, or if they were taking medications at the time of enrollment. Informed consent was obtained from each subject before this study. The Institutional Review Board of the University of Fukui approved this study.

Details of the procedure are as follows: amniotic fluid samples of $15 \mathrm{~mL}$ were obtained using amniocenteses; the samples were fractioned by differential centrifugation according to the methods described by Hook et al. (3). Each amniotic fluid sample was centrifuged at $370 \times g$ for $10 \mathrm{~min}$ to remove the sediment, which consisted mainly of cells. Subsequently, the supernatant was centrifuged at $9200 \times g$ for $20 \mathrm{~min}$. The $9200 \times g$ supernatants were maintained at $37^{\circ} \mathrm{C}$ until analysis of micelles.

Analysis of micelles. All samples for electron microscopy were stained negatively using $2 \%$ phosphotungstic acid at $\mathrm{pH} 7.2(13,14)$ and analyzed within $1 \mathrm{~h}$ after sample preparation. Electron micrographs were viewed at 75 $\mathrm{kV}$ using an electron microscope (H-7100 FA; Hitachi, Tokyo, Japan).

Identification of SP-B in the micelle fraction of term amniotic fluid. The presence of SP-B in the micelle fraction was examined by using Western blot analysis (15). Briefly, $15 \mathrm{~mL}$ of the $9200 \times g$ supernatant sample retrieved from term pregnancy was centrifuged at $22,500 \times g$ for $30 \mathrm{~min}\left(4^{\circ} \mathrm{C}\right)$. Half of the resultant pellet was dispersed with $100 \mu \mathrm{L}$ of saline and maintained at $4^{\circ} \mathrm{C}$ until analysis of micelles. The remaining pellet was used for Western blot analysis: solubilized in $60 \mu \mathrm{L}$ of gel sample buffer, boiled for $5 \mathrm{~min}$, and separated using a 15\% acrylamide SDS-PAGE gel. Electrophoresed samples were transferred to a nitrocellulose membrane (Trans-Blot Transfer Medium; Bio-Rad, Hercules, CA) following the instructions provided by the manufacturer. Immunoblotting was performed using a horseradish peroxidase system (Santa Cruz Biotechnology, Santa Cruz, CA) and bands were visualized by enhanced chemiluminescence according to the ECL Western Blotting Detection System (Amersham, Little Chalfont, Buckinghamshire, UK). A 1:5000 dilution of mouse anti-human SP-B MAb (clone $1 \mathrm{BQ}$, subclass $\mathrm{IgG}_{2 \mathrm{~b}}$; Chemicon International, Temecula, CA) was used as the primary antibody. The secondary antibody consisted of a 1:2000 dilution of horseradish peroxidase-conjugated goat antimouse IgG (Santa Cruz Biotechnology).

Turbidity assay of mixtures of vernix caseosa and amniotic fluid micelles. Amniotic fluid samples of $15 \mathrm{~mL}$ were obtained by amniocenteses from four healthy Japanese pregnant women at term. The samples were methodically fractioned by differential centrifugation $(3,15)$. The $22,500 \times g$ sediments (micelle fractions) were dispersed with $1 \mathrm{~mL}$ of saline and subsequently added to each 1.5 -mL polypropylene microfuge tube, interiorly coated with $10 \mathrm{mg}$ of vernix caseosa (5). The samples from the vernix caseosa-saline mixtures $(n=4)$ were used as controls.

Each sample was analyzed spectrophotometrically at $650 \mathrm{~nm}$ before and after overnight incubation at $37^{\circ} \mathrm{C}(5)$. The $\mathrm{OD}$ correlated with solution turbidity that resulted from detachment of vernix from the walls of the microfuge tubes (5). Changes in absorbance values of micelle samples during overnight incubation were compared with those of control samples.

Statistical analysis. Data are expressed as mean \pm SD. Statistical analysis was performed using StatView software, version 5.0 (SAS Institute, Cary, $\mathrm{NC})$. The $t$ test was used for continuous variables. All statistical tests were two-sided and significance was defined as $p<0.05$.

\section{RESULTS}

The CMC value of surfactant TA obtained from the concentration/surface-tension curve was $80 \mu \mathrm{g} / \mathrm{mL}$ (Fig. 1). The value is converted to approximately $67 \mu \mathrm{g} / \mathrm{mL}$ in phospholipid concentration (10). Surfactant TA formed micelles above its CMC at phospholipid concentrations of 75, 100, 150 (Fig. $2 A$ ), 200, and $500 \mu \mathrm{g} / \mathrm{mL}$.

Vernix detached from the tube wall above the CMC of surfactant TA. Surfactant TA interacted with vernix caseosa and subsequently formed micelles (Fig. 2B).

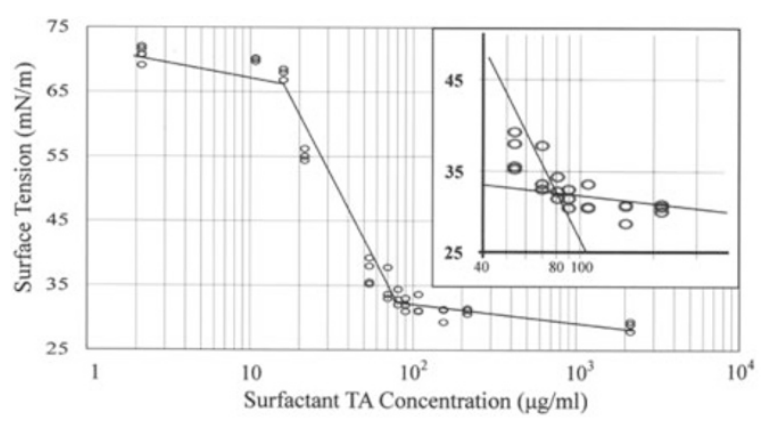

Figure 1. Surface tension behavior of surfactant TA solutions. The inset shows a magnified image of the region of interest. Surface tension decreased from 70.8 to $28.8 \mathrm{mN} / \mathrm{m}$ with increasing concentration of surfactant TA. The $\mathrm{CMC}$ represented the concentration at the intersection of the two lines of the concentration/surface-tension curve. The CMC value was $80 \mu \mathrm{g} / \mathrm{mL}$.

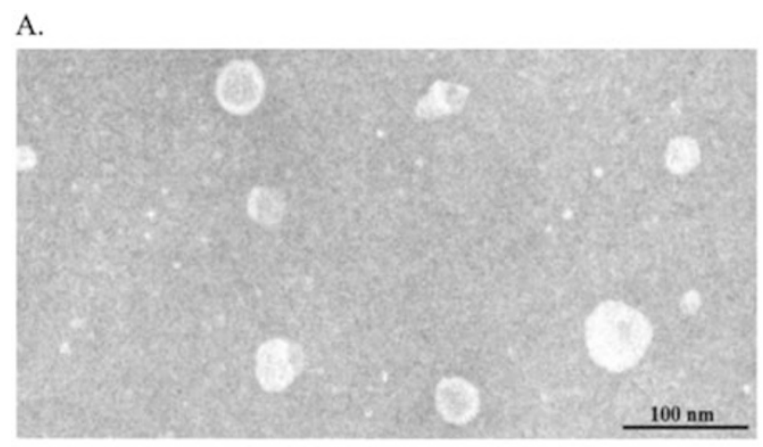

B.
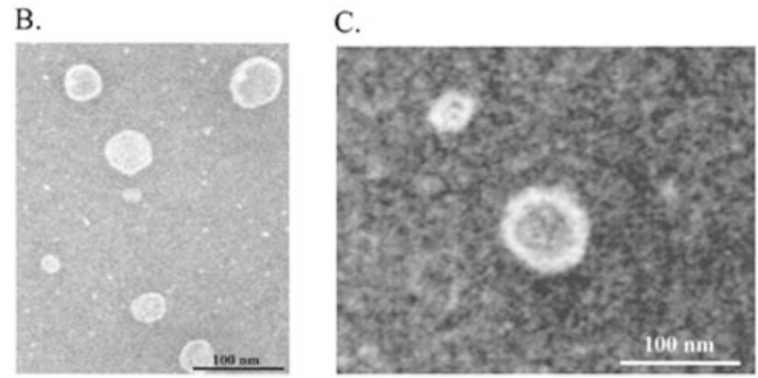

Figure 2. Negative stain electron micrograph of micelles. (A) Micrograph of surfactant TA solution at phospholipid concentration of $150 \mu \mathrm{g} / \mathrm{mL}$. Micelles are visible against the stained background. The diameter of spheroidal and rod-shaped micelles ranges from 10 to $70 \mathrm{~nm}$. The average diameter of micelles derived from surfactant TA is $35.4 \mathrm{~nm}$. (B) Micrograph of mixtures of vernix caseosa and surfactant TA. Micelles are visible similarly to surfactant TA solution $(A)$. The average diameter of micelles derived from the mixtures is $46.8 \mathrm{~nm}$, which was not significantly different from the average diameter of surfactant TA micelles $(p>0.05)$. $(C)$ Micrograph of human amniotic fluid at term. Despite their lower number, micelles are similar to surfactant TA solution $(A$ and $B$ ). Calibration bar: $100 \mathrm{~nm}$; original magnification: $\times 200,000$.

Micelles were also detected in all but one $9200 \times g$ supernatants of human full-term amniotic fluid samples (Fig. $2 C$ ). In addition, Western blot analysis revealed the presence of SP-B in the micelle fraction of term amniotic fluid (Fig. 3, $A$ and $B$ ). However, no micelles were identified in human amniotic fluid samples harvested at 16 wk of gestation.

Amniotic fluid micelles interacted with vernix caseosa and subsequently induced vernix detachment from the tube wall (Fig. 4). In contrast, for control samples, the mixtures of vernix caseosa and physiologic saline remained clear after overnight incubation. The increasing absorbance values at 650 
A.

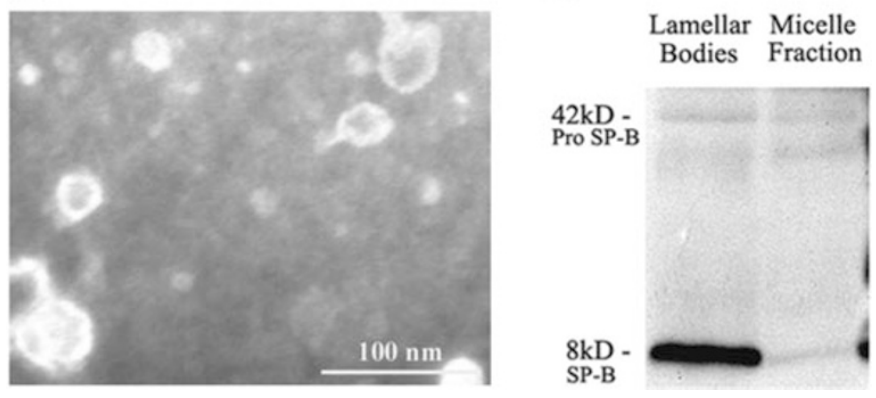

Figure 3. Identification of SP-B in the micelle fraction of full-term amniotic fluid. (A) Negative stain electron micrograph of 22,500 $\times g$ sediments retrieved from human amniotic fluid at term. Micelle particles are visible (see Fig. 2). Calibration bar: $100 \mathrm{~nm}$; original magnification: $\times 200,000$. $(B)$ Western blot analysis revealed $42-\mathrm{kD}$ pro-SP-B and $8-\mathrm{kD}$ mature SP-B in the micelle fraction of term amniotic fluid. The fraction of the lamellar bodies, which is rich in SP-B, was used as positive control.

$\mathrm{nm}$ for micelle samples and control samples were $0.496 \pm$ 0.178 and $0.055 \pm 0.017$, respectively $(p=0.0027)$.

\section{DISCUSSION}

The major finding of our study was the presence of micelles in human full-term amniotic fluid. Narendran et al. (5) speculated that amniotic fluid turbidity is secondary to roll-up detachment of vernix caseosa induced by pulmonary surfactant. The roll-up phenomenon is intrinsically controlled by micellization process kinetics; the detergent aqueous solution starts to remove contaminants at or near the CMC and reach maximum detergency at concentrations greater than the CMC $(6,7)$. Moreover, micelle induces the dissolution of the mass contaminant from surfaces directly into the aqueous phase (6). The present study also showed that human amniotic fluid micelles induce detachment of vernix caseosa under in vitro conditions. Our results provide a strong support for the hypothesis proposed by Narendran et al. (5).

Micelle particles in term amniotic fluids are similar to the micelles derived from surfactant TA. We also demonstrated the association of SP-B with micelles of human amniotic fluid. SP-B is important for optimizing the surface active properties of pulmonary surfactant and is co-secreted with the surfactant (2). Accordingly, our results suggest that amniotic fluid micelles are associated with pulmonary surfactant.

Secretion of pulmonary surfactant begins at 25-30 wk gestation $(1,2)$. In addition, detachment of the vernix caseosa from fetal skin into the amniotic fluid commences at $34 \mathrm{wk}$ gestation (4). These chronological events fit with the results of the present study of the absence of micelles in all amniotic fluid samples taken at $16 \mathrm{wk}$ of gestation. The absence of micelles in amniotic fluid from 16-wk pregnancies is a further indication that pulmonary surfactant is associated with the micelles in the full-term amniotic fluid. Micelles were not detected at term in one of our six amniotic fluid samples. Thus, the latter case points to the limitation of our procedure.

Numerous studies have reported that the surface-active fractions in amniotic fluid originate from fetal lung $(2,3)$. Hook et al. (3) indicated that the lamellar bodies in amniotic
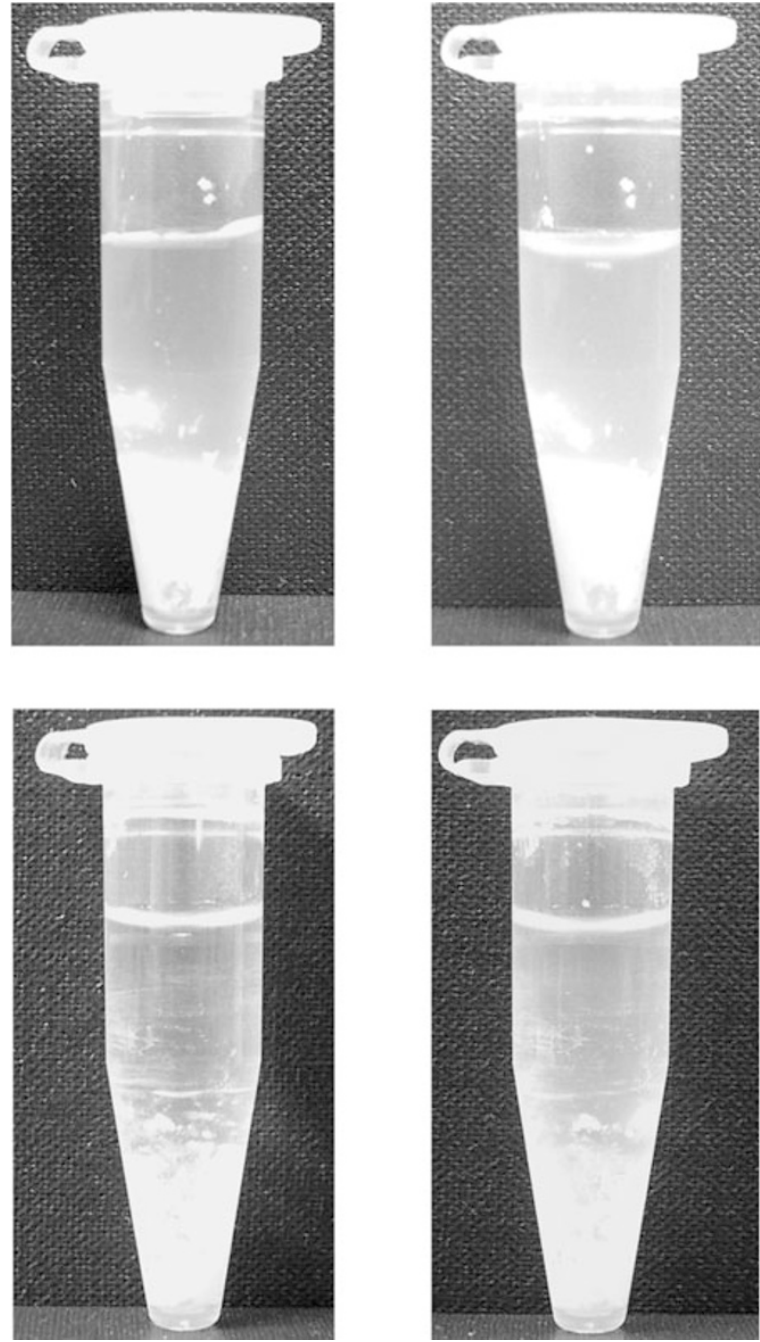

Figure 4. Series of images showing time-dependent detachment of vernix caseosa by amniotic fluid micelles (top) and physiologic saline (bottom). The turbidity of vernix caseosa-amniotic fluid micelle samples increased significantly during overnight incubation at $37^{\circ} \mathrm{C}$ (top left, preincubation; top right, postincubation). In contrast, the mixtures of vernix caseosa and physiologic saline remained clear after overnight incubation (bottom left, preincubation; bottom right, postincubation).

fluid are pulmonary surfactants based on differential centrifugation of the amniotic fluid. Lamellar bodies containing concentric whorls of phospholipids are recognized as storage forms of pulmonary surfactant $(2,3,16)$. However, Hook's procedure is unsuitable for analysis of small lipid vesicles. Furthermore, the lamellar bodies range in size from 1 to $5 \mu \mathrm{m}$ (3); they are sufficiently large to obscure micelles (10-70 nm). On the other hand, a negative staining method is useful for examining suspensions of small particles, including micelles $(13,14)$. Using such technique, we were able to identify the presence of micelles in the $9200 \times g$ supernatant fraction of human full-term amniotic fluid, which differed from the fraction of lamellar bodies. This finding suggests that micelles are not derived from lamellar bodies during the preparation of amniotic fluid sample. Rather, micelles are naturally present in human amniotic fluid.

Micellization is an important step for postnatal lipid absorption because fat is insoluble in the aqueous environment of the 
small-intestinal lumen $(1,8)$. Micelles exist in amniotic fluid swallowed by the fetus and in human breast milk ingested by neonates. Numerous studies have shown that maturation of the fetal gastrointestinal tract is partially enhanced by swallowed amniotic fluid $(1,17)$. The fetal lung synthesizes and secretes much greater amounts of surfactant than are needed for air adaptation after birth. The micelles in swallowed amniotic fluid might act as a promoter of fetal intestinal maturation.

Visualization of the micelle particles in human amniotic fluid has not been reported previously. Our results support the view that pulmonary surfactant could induce the detachment of vernix caseosa, thus increasing the turbidity of the amniotic fluid. Although further studies are needed, our findings enhance our understanding of the physiologic interaction between lung development and skin development.

Acknowledgments. The authors thank Hitoshi Takagi, Shintaro Nishijima, Mikiko Misawa, and Mari Ito for the excellent technical assistance.

\section{REFERENCES}

1. Avery GB, Fletcher MA, MacDonald MG 1999 Neonatology: Pathophysiology and Management of the Newborn. 5th Ed. Lippincott Williams \& Wilkins, Philadelphia, $\mathrm{pp}$

2. Notter RH 2000 Lung Surfactants: Basic Science and Clinical Applications. Marcel Dekker, Inc., New York, pp
3. Hook GE, Gilmore LB, Tombropoulos EG, Fabro SE 1978 Fetal lung lamellar bodies in human amniotic fluid. Am Rev Respir Dis 117:541-550

4. Agorastos T, Lamberti G, Vlassis G, Zournatzi B, Papaloucas A, 1986 Methods of prenatal determination of fetal maturity based on differentiation of the fetal skin during the last weeks of pregnancy. Eur J Obstet Gynecol Reprod Biol 22:29-40

5. Narendran V, Wickett RR, Pickens WL, Hoath SB 2000 Interaction between pulmonary surfactant and vernix: a potential mechanism for induction of amniotic fluid turbidity. Pediatr Res 48:120-124

6. Kabin JA, Tolstedt SL, Saez AE, Grant CS, Carbonell RG 1998 Removal of organic films from rotating disks using aqueous solutions of nonionic surfactants: effect of surfactant molecular structure. J Colloid Interface Sci 206:102-111

7. Lai K-Y, McCandlish EF, Aszman H 1996 Light duty liquid detergents. In: Lai K-Y (ed) Liquid Detergents (Surfactant Series/67). Marcel Dekker Inc., New York, pp 207-259.

8. Murray RK, Granner DK, Mayes PA, Rodwell VW 2000 Harper's Biochemistry. 25th Ed. Appleton \& Lange, Stamford, Connecticut, pp

9. Fujiwara T, Maeta H, Chida S, Morita T, Watabe Y, Abe T 1980 Artificial surfactant therapy in hyaline-membrane disease. Lancet 1:55-59

10. Tanaka Y, Takei T, Kanazawa Y, Seida K, Masuda K, Kiuchi A, Fujiwara T 1982 Preparation of surfactant from minced bovine lung, chemical composition and surface properties. J Jpn Med Soc Biol Interface 13:87-94

11. Castro M, Ritacco H, Kovensky J, Fernandez-Cirelli A, 2001 A simplified method for the determination of critical micelle concentration. J Chem Educ 78:347-348

12. Ananthapadmanabhan KP, Goddard ED, Turro NJ, Kuo PL 1985 Fluorescence probes for critical micelle concentration. Langmuir 1:352-355

13. Maggio B, Albert J, Yu RK 1988 Thermodynamic-geometric correlations for the morphology of self-assembled structures of glycosphingolipids and their mixtures with dipalmitoylphosphatidylcholine. Biochim Biophys Acta 945:145-160

14. Dubertret B, Skourides P, Norris DJ, Noireaux V, Brivanlou AH, Libchaber A, 2002 In vivo imaging of quantum dots encapsulated in phospholipid micelles. Science 298:1759-1762

15. Glumoff V, Vayrynen O, Kangas T, Hallman M 2000 Degree of lung maturity determines the direction of the interleukin-1-induced effect on the expression of surfactant proteins. Am J Respir Cell Mol Biol 22:280-288

16. Wright JR 1990 Clearance and recycling of pulmonary surfactant. Am J Physio 259:L1-L12

17. Ross MG, Nijland MJ 1998 Development of ingestive behavior. Am J Physiol 274:R879-R893 